\title{
NeuroRegulation
}

\section{Toward Development of Sham Protocols for High- Definition Transcranial Direct Current Stimulation (HD-tDCS)}

\author{
Jessica D. Richardson ${ }^{1 *}$, Paul Fillmore ${ }^{1}$, Abhishek Datta ${ }^{2}$, Dennis Truong ${ }^{3}$, \\ Marom Bikson ${ }^{3}$, Julius Fridriksson ${ }^{1}$ \\ ${ }^{1}$ Department of Communication Sciences and Disorders, The University of South Carolina, USA \\ ${ }^{2}$ Soterix Medical Inc., New York, New York, USA \\ ${ }^{3}$ Department of Biomedical Engineering, The City College of New York of CUNY, USA
}

*Address correspondence to: Jessica D. Richardson, Ph.D., Department of Communication Sciences \& Disorders, University of South Carolina, Discovery 1, 915 Greene Street, Columbia, SC 29208. Email: j.d.richardson@sc.edu

Copyright: (C) 2014 Richardson et al. This is an Open Access article distributed under the terms of the Creative Commons Attribution License (CC-BY).

Author Note: This work was supported by the following grants: Wallace $\mathrm{H}$. Coulter Foundation (MB), NIH/NIDCD grant DC011739 (JF).

\begin{abstract}
High-definition transcranial direct current stimulation (HD-tDCS) is a noninvasive cortical stimulation (NICS) technique that, due to the utilization of multi-electrode stimulation, may enable development of sham conditions characterized by indistinguishable scalp sensations compared to active conditions, with little or no cortical influence. We sought to contribute to the development of an optimal sham electrode configuration for HD-tDCS protocols by gathering ratings of overall sensation reported by participants during different electrode configurations and current intensities. Twenty healthy participants completed a magnitude estimation task during which they rated their "overall sensation" in 1-minute intervals during five 5-minute stimulation conditions. A $5 \times 5$ (Time $\times$ Stimulation condition) analysis of variance (ANOVA) was conducted to determine if sensation measurements differed over time, and how this varied by condition. Null hypothesis significance tests and equivalence tests were conducted to determine which sham conditions were statistically indistinguishable from the experimental condition. The ANOVA revealed main effects for Time and Stimulation condition. Planned comparisons, comparing each sham condition to the experimental condition (4x1 ring configuration, $2 \mathrm{~mA}$ ), revealed differences in sensation ratings for all but one condition (Sham $1 \times 1 \mathrm{~A}$ ); no sham conditions were found to be statistically equivalent to the experimental condition. Our HD-tDCS findings build upon previous NICS reports of differences in sensation ratings between sham versus experimental conditions when traditional "ramping down" approaches were used. Alternative multi-electrode configurations that manipulate electrode placement to shunt current across the scalp warrant further investigation as valid blinding methods.
\end{abstract}

Keywords: HD-tDCS; sham; sensation; tDCS 


\section{Introduction}

Noninvasive cortical stimulation (NICS) techniques, such as transcranial magnetic stimulation (TMS) and transcranial direct current stimulation (tDCS), are useful for investigating brain-behavior relationships in healthy populations and have also begun to be used therapeutically in clinical populations (Brunoni et al., 2012; Fregni \& Pascual-Leone, 2007; Williams, Imamura, \& Fregni, 2009). TMS directly alters cortical excitability via application of a magnetic stimulus to the scalp that travels through overlying matter to influence discrete cortical areas. Depending largely upon temporal patterning and stimulus intensity, cortical effects can be inhibitory or excitatory (Chrysikou \& Hamilton, 2011; Vallence \& Ridding, 2013). tDCS modulates cortical excitability via application of a weak electrical stimulus to the scalp (1-2 mA) through two surface electrodes. The weak stimulus travels through overlying matter to diffusely and indirectly influence cortical excitability. Much remains to be learned about dose-response relationships for tDCS, but in general the area under the cathodal surface electrode is more inhibitory and the area under the anodal surface electrode is more excitatory (Chrysikou \& Hamilton, 2011; Vallence \& Ridding, 2013).

Just as placebo trials are fundamental for proving drug effectiveness in pharmacological research, the ability to blind both experimenters and subjects to stimulation condition is important for unbiased interpretation of NICS results and is accomplished via "sham" forms of NICS. Pharmacological investigations often employ an active placebo (to induce side effects in absence of target effect) to avoid unintended unblinding of participants by absence of side effects (e.g., Moncrieff, Wessely, \& Hardy, 2004). Similarly, TMS investigations often utilize active sham conditions (e.g., change in angle of coil orientation with unchanged or reduced stimulus intensity) that produce comparable scalp sensations with reduced cortical effect (e.g., Deng \& Peterchev, 2011; Loo et al., 2000). tDCS protocols generally utilize the "fade in - short stimulation - fade out" approach, where the current is ramped down following a brief period of delivery designed to induce initial sensations that are thought to fade (Gandiga, Hummel, \& Cohen, 2006). In fewer instances, active control conditions (e.g., current delivery to cortical area thought to be unimportant to experimental task; Boggio et al., 2008) or low-current conditions (e.g., $0.1 \mathrm{~mA}$; Coffman, Trumbo, \& Clark, 2012) are utilized for comparison.

It is uncertain that subjects are truly blinded during sham conditions using current NICS techniques. Recent investigations of sham TMS revealed that a greater proportion of subjects in active experimental groups guess correctly which condition they received (Broadbent et al., 2011), and special care must be taken when designing tDCS trials (e.g., selection and preparation of electrodes that determine sensation in the active phase; Minhas, Datta \& Bikson, 2011; Dundas, Thickbroom, \& Mastaglia, 2007) to avoid significant differences in sensory side effects and severity between experimental and sham tDCS conditions (e.g., Kessler, Turkeltaub, Benson, \& Hamilton, 2012). High-definition tDCS (HDtDCS) is a new NICS technique that improves current focality and intensity using multiple gel-based electrodes, similar to those used in electroencephalography (EEG), to deliver electrical stimulation (Datta et al., 2009; Dmochowski, Datta, Bikson, Su, \& Parra, 2011). The parameters for an acceptable sham HD-tDCS are being explored (Borckardt et al., 2012). With HD-tDCS, it is possible to manipulate electrode configuration to purposefully shunt current across the scalp. This could facilitate development of active sham conditions whereby current is continually applied and resultant scalp sensations are indistinguishable from active experimental conditions, with little or no cortical modulation. The purpose of this study is to contribute to the development of an optimal sham condition for HD-tDCS 
protocols by gathering detailed ratings of sensations experienced by participants during different electrode configurations and current intensities.

\section{Materials and Methods}

\section{Subjects}

Twenty healthy participants between the ages of 18 and 75 years $(M=30.3$ years; 9 females) participated in this experiment. The University of South Carolina Institutional Review Board approved this study.

\section{HD-tDCS}

Stimulation was delivered using High-Definition electrode insets (model HD2, Soterix Medical, Inc.) that are safe and well tolerated for currents up to $2.0 \mathrm{~mA}$ (Borckardt et al., 2012; Minhas et al., 2010; Villamar et al., 2013). Prior to electrode placement, a mild anesthetic (1-2 $\mathrm{mL}$ Lanacane, active ingredient $6 \%$ benzocaine) was applied to the scalp under HD-insets to reduce scalp irritation and sensations. Sintered $\mathrm{Ag} / \mathrm{AgCl}$ electrodes were then immersed in conductive jelly (Signa gel $\AA_{\text {, }}$ Parker Laboratories) inside the insets. 1.0 to 2.0 mA HD-tDCS was administered via a battery-powered constant current stimulator that was connected to the electrodes through a Multi-Channel Stimulation Adapter (Soterix Medical, Inc.).

There were five conditions with two electrode montages for this study. Each condition was 5 minutes in duration. To guard against order effects, partial counterbalancing was employed, and conditions were administered in random order to each participant with at least a 1minute break between each condition. Three conditions involved the $4 \times 1$ ring montage (Figure 1a), with the cathode electrode centered over the left inferior parietal lobe and 4 anode electrode returns circling the target region: (a) Exp4x1 - experimental condition, subjects received 5 minutes of $2.0 \mathrm{~mA}$ - this montage was selected because it has been modeled and used in clinical research to provide focal cortical stimulation (e.g., Datta et al., 2009; Borckardt et al., 2012; Villamar et al., 2013); (b) Sham4x1A - 45 seconds of $2.0 \mathrm{~mA}$ ramped down to $1.0 \mathrm{~mA}$ for the remaining time (active fade sham); and (c) Sham4x1B - 45 seconds of $2.0 \mathrm{~mA}$ ramped down to zero current for the remaining time (inert fade sham). The two remaining conditions involved an active sham, using the 1x1 montage (Figure 1b) where the anode and cathode electrodes were placed immediately adjacent to the other in order to shunt at least part of the current across the scalp: (a) Sham1x1A - 5 minutes of 2.0 $\mathrm{mA}$, and (b) Sham1x1B - 5 minutes of $1.5 \mathrm{~mA}$. Pilot testing revealed near floor ratings for sensation for $1 \times 1$ at $1.0 \mathrm{~mA}$, so this was not pursued as a viable sham option for our experimental montage (see Discussion). Participants did not perform tasks during HD-tDCS administration.

The experimental and sham montages (electrode positions and current applied) were modeled in a single individual using methods described previously (Datta et al., 2009). Current density at the scalp (reflecting sensation) and electric field at the cortex (reflecting neuromodulation) were predicted for both $4 \times 1$ and $1 \times 1$ configurations, and are illustrated in Figure 1 ( $a$ and $b)$. 

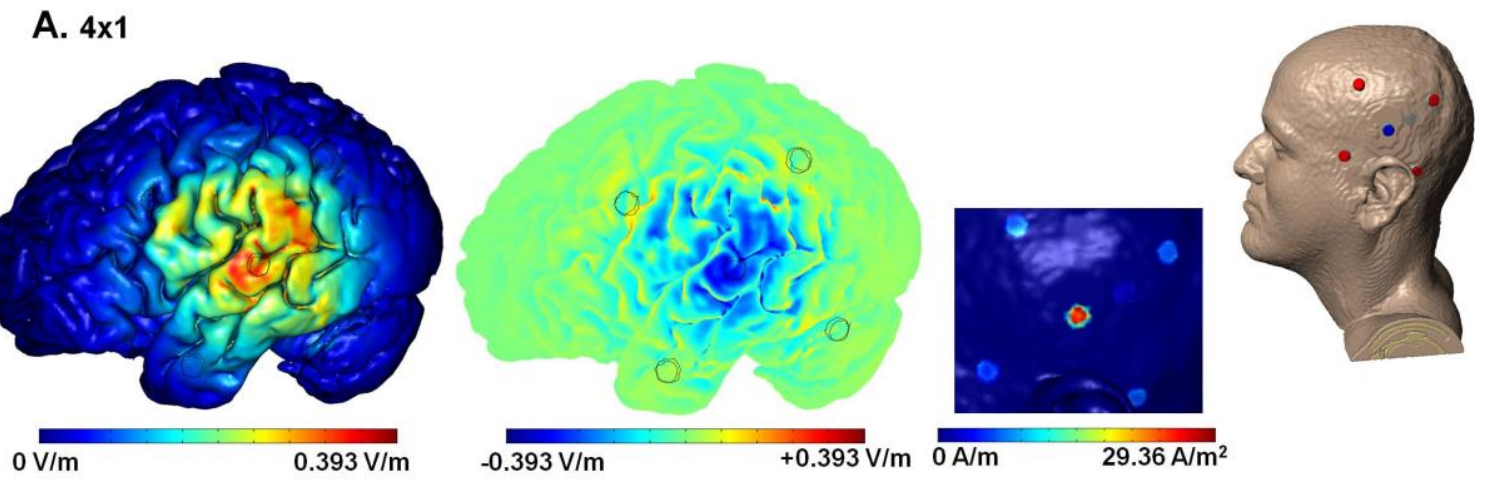

\section{B. SHAM (1x1)}
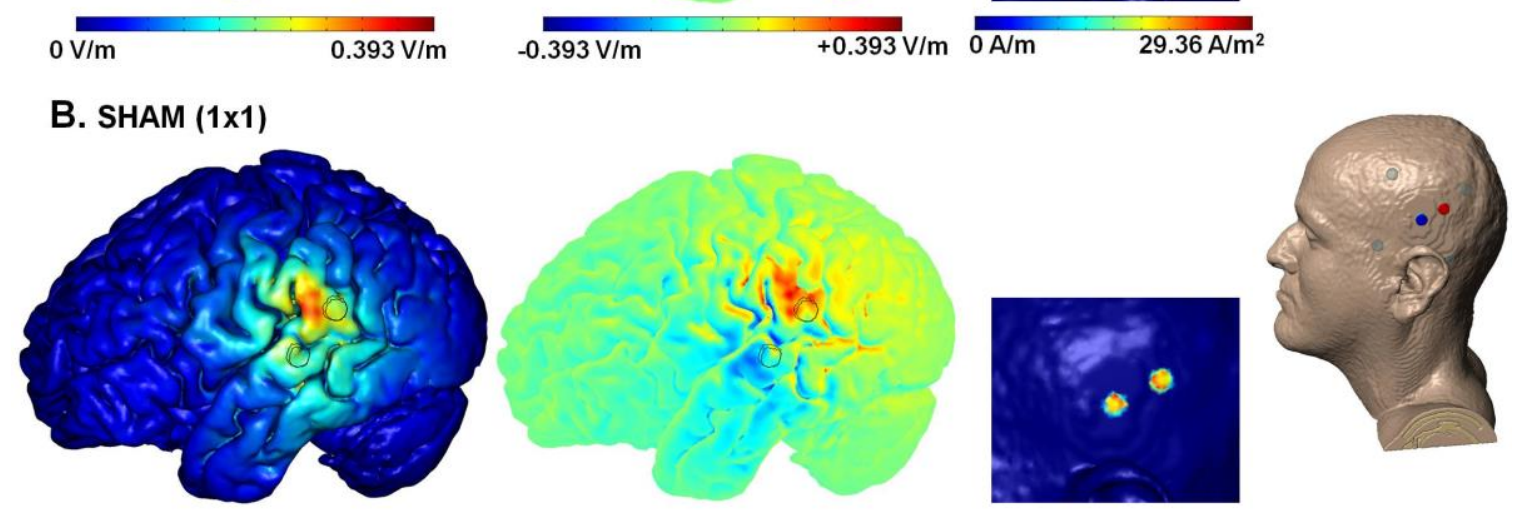

\section{SHAM (3x1)}
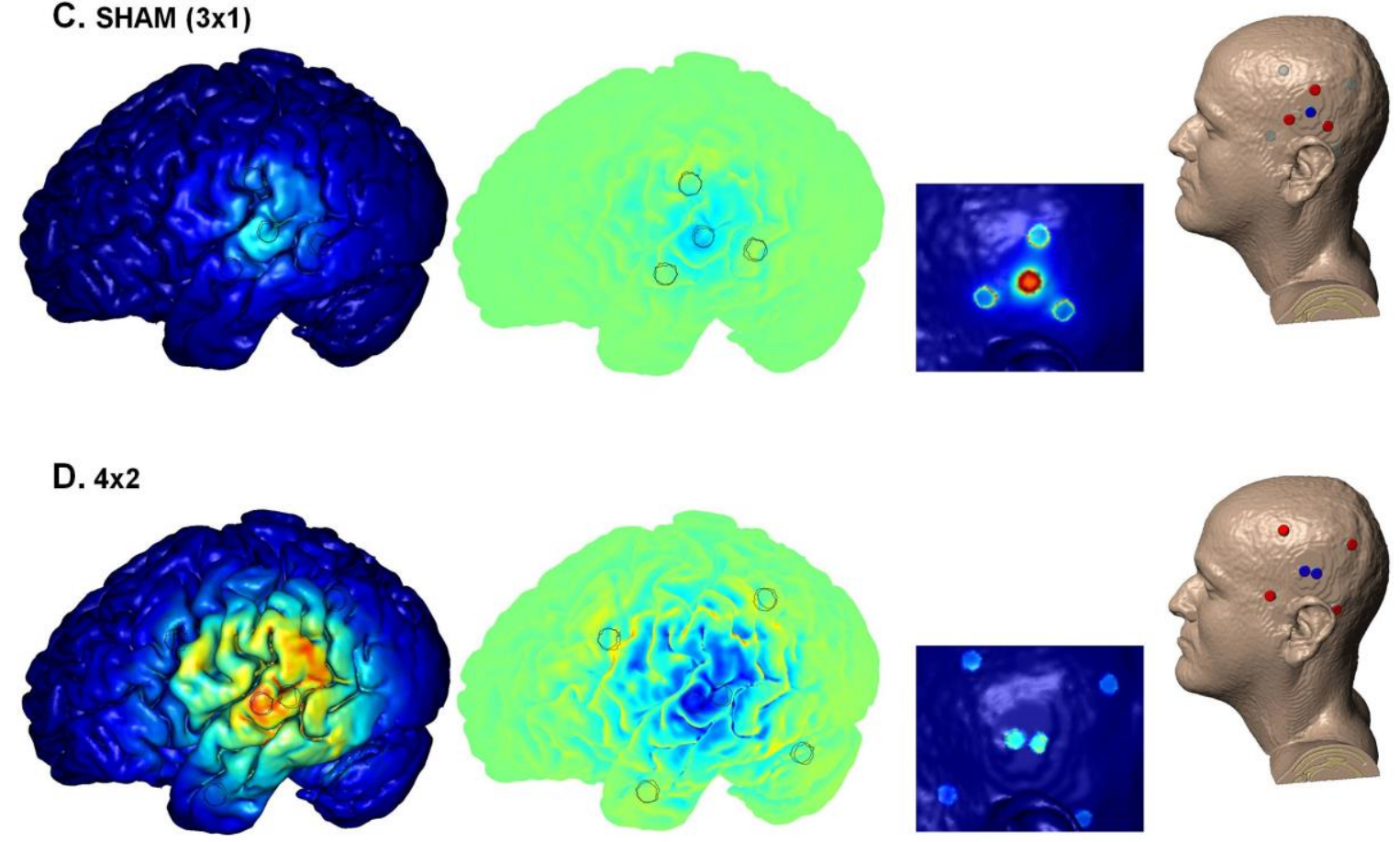

Figure 1. High-resolution $1 \mathrm{~mm} 3 \mathrm{MRI}$-derived FEM simulations of current flow using 4 electrode montages. Cortical electric field magnitude (1st panel), radial cortical electric field (which considers inward/outward flow; 2nd panel), and current density at the skin (indicative 
of sensation; 3rd panel) for each montage (4th panel) are considered. The colored circles on the rendered image (4th panel) indicate the status of the electrode in the loaded cap: blue = cathode, red = anode, gray = inactive. A) The control experimental montage, 4x1 HD-tDCS with $2 \mathrm{~mA}$ applied through the center cathode, was used experimentally and in the simulation as a standard for comparison. B) The proximal Sham $1 \times 1$ montage, with $2 \mathrm{~mA}$, resulted in reduced, but not negligible, cortical current flow and moderately reduced skin current density compared to the control case, which is consistent with experimental findings. C) The proximal Sham $3 \times 1$ montage, with $2 \mathrm{~mA}$, resulted in maximal skin shunting as indicated by low brain electric field, but skin current density comparable to the control case, suggesting this montage should be further evaluated as an active sham. D) The 4x2 HD-tDCS montage with $1 \mathrm{~mA}$ current applied through each of the center cathodes ( $2 \mathrm{~mA}$ total) results in comparable electric field as the control case, but significantly reduced current density. This result is consistent with preliminary findings that stimulation with up to $1 \mathrm{~mA}$ per electrode approaches sensation floor for most subjects, such that the $4 \times 2$ HD-tDCS montage may be explored as a new test condition with no active (current flow) sham required.

\section{Sensation Ratings}

Participants completed a magnitude estimation task (no modulus) during which they rated "overall sensation" in 1-minute intervals during each condition. A left-to-right visual analog scale was used, ranging from "no sensation" to "maximum sensation". Participants were instructed to make subsequent ratings relative to the first rating. To ensure accurate rating at designated time points, only overall sensation was assessed rather than requiring subjects to track multiple sensations (which can be subjective, difficult to disentangle, and not experienced by every participant). All ratings were scaled as within-subject in reference to the individual maximum across time and condition (rating at each time/maximum rating for that participant at any time, in any condition) to allow for cross-subject comparisons, given differences in individual sensitivity.

\section{Results}

A $5 \times 5$ (Time $x$ Stimulation condition) analysis of variance (ANOVA) was conducted to determine if sensation measurements differed over time and/or by condition. There was a main effect for Time, $F(4,76)=33.738, p<.001$, and Stimulation condition, $F(4,76)=5.576$, $p=.001$. The interaction effect was not significant $(p=.063)$. See Figures $2 \mathrm{a}$ and $2 \mathrm{~b}$ for sensation mean and standard deviation for each condition. A $5 \times 5$ (Time $\times$ Order) ANOVA did not reveal main effects for order $(p=.511)$ nor an interaction $(p=.128)$.

Planned paired samples t-tests were performed for each time point to determine which sham conditions were significantly different from Exp4x1. Significant differences (Holm-Bonferroni corrected at each time point) in the following sham conditions compared to the Exp4x1 were observed: Sham 1x1B at time points 1 and $3(p=.012, .005)$, Sham $4 \times 1 \mathrm{~A}$ at time points 3 through $5(p=.006, .004, .008)$ and Sham $4 \times 1 \mathrm{~B}$ at time points 2 through $5(p<.001)$. Sham $1 \times 1 A$ showed no significant differences at any time point. Effect sizes (Cohen's adjusted $d$ ) were calculated for each comparison and are displayed in Figure 2c; most effect sizes (13 of 20 comparisons) are medium to large. 

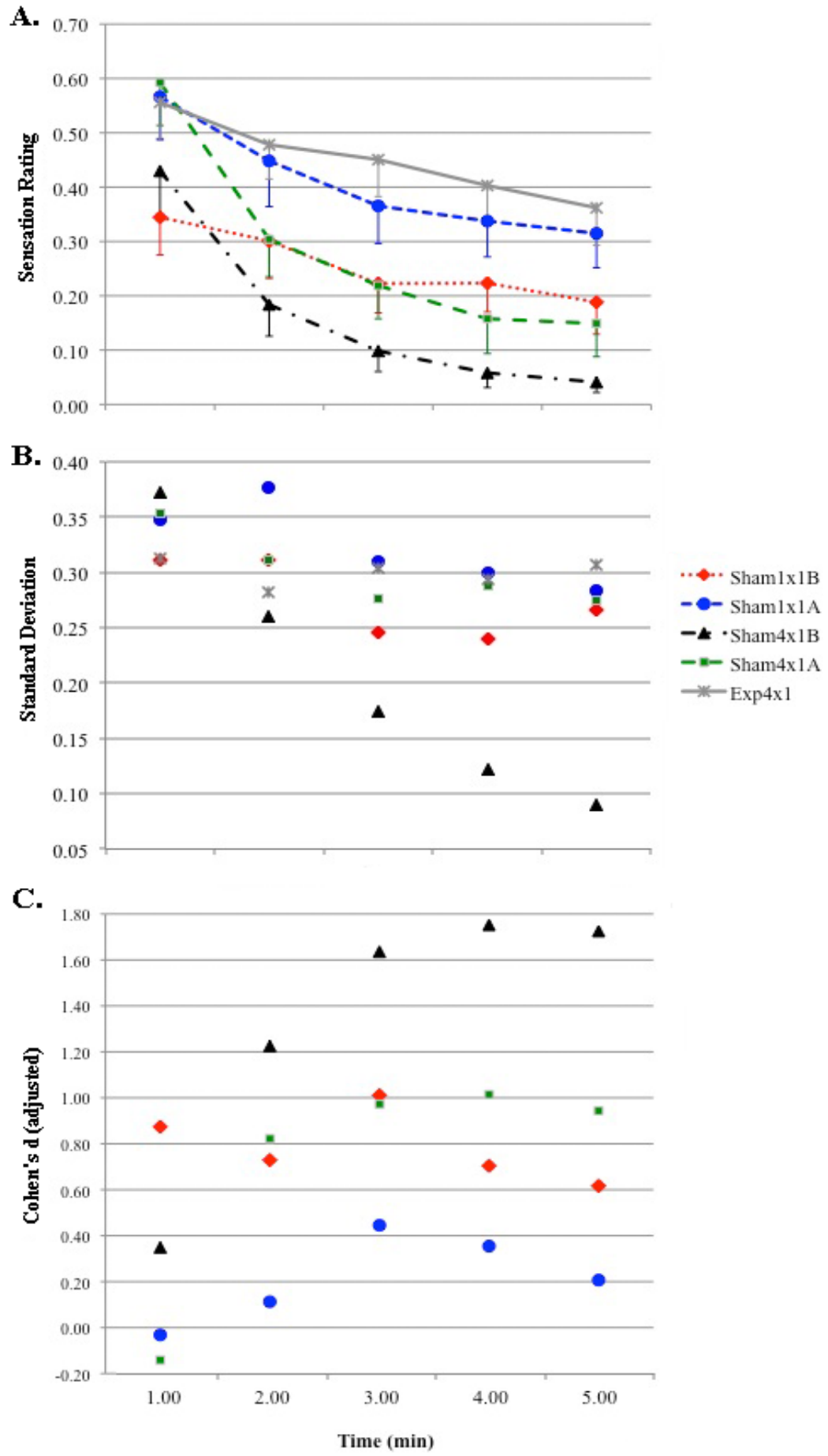

Figure 2. A) Sensation curves (created with average ratio values) per condition. The x-axis represents time; the $y$-axis represents sensation ratio measurement. B) Standard deviation of mean ratio values per condition. The $x$-axis represents time; the $y$-axis represents standard deviation. C) Effect sizes for each sham-to-experimental condition comparison. The $x$-axis represents time; the y-axis represents Cohen's adjusted $d$. 
Equivalence testing utilizing the confidence interval approach (Tryon, 2001; Rusticus \& Lovato, 2011) was performed to determine which sham conditions could be considered equivalent to the experimental condition. The a priori equivalence interval $(l$; mean $+/-0.5$ $S D$ ) was established to identify the boundaries of a range of values that might indicate a practically significant difference. The $90 \%$ confidence intervals $(\mathrm{Cls})$ from the ANOVA were used to define the maximum probable difference (MPD) between the two means. Thus, mean differences were classified as: within I (statistically equivalent), partially overlapping I (statistically indeterminate), or outside I (statistically non-equivalent). No MPDs at any time point were classified as equivalent (see Table 1). MPDs for Sham4x1B at time points 2 through 5 were statistically non-equivalent, and all other comparisons were statistically indeterminate.

Table 1

Equivalence Test Results for Paired Differences at Each Time Point

\begin{tabular}{|c|c|c|c|c|c|c|c|c|c|c|}
\hline \multirow{5}{*}{ Exp4x1 } & Time & (T1) & $T$ & 2 & & & $T$ & & & \\
\hline & \multicolumn{10}{|c|}{ Equivalence Interval $(I)$} \\
\hline & \multicolumn{2}{|c|}{$+/-.1566$} & \multicolumn{2}{|c|}{$+/-.1410$} & \multicolumn{2}{|c|}{$+/-.1522$} & \multicolumn{2}{|c|}{$+/-.1466$} & \multicolumn{2}{|c|}{$+/-.1536$} \\
\hline & \multicolumn{10}{|c|}{ 90\% Confidence Intervals of Paired Differences (paired with Exp4x1) } \\
\hline & Lower & Upper & $\mathrm{L}$ & $\mathrm{U}$ & $\mathrm{L}$ & $\mathrm{U}$ & $\mathrm{L}$ & $\mathrm{U}$ & $\mathrm{L}$ & $\mathrm{U}$ \\
\hline Sham $4 \times 1 A$ & -.179 & .106 & .058 & .290 & .101 & .362 & .116 & .375 & .089 & .337 \\
\hline Sham 4x1B & -.071 & .324 & $.163 *$ & $.426^{*}$ & $.232^{*}$ & $.473 *$ & $.235^{*}$ & $.455^{*}$ & $.208^{*}$ & $.433^{*}$ \\
\hline Sham1x1A & -.185 & .164 & -.117 & .176 & -.020 & .190 & -.034 & .164 & -.078 & .172 \\
\hline Sham1x1B & .079 & .344 & .044 & .310 & .104 & .352 & .043 & .316 & .020 & .328 \\
\hline
\end{tabular}

Note. The equivalence interval $(I)$ is $+/$ - half the SD of the mean for Exp4x1 at each time point. The $90 \%$ confidence intervals (lower to upper bounds) of the paired differences are presented as the maximum probable difference (MPD).

\section{Discussion}

The essential challenge with shamming all NICS is that energy delivered to the brain must pass, typically at higher intensity, through the scalp. Traditional sham approaches reduce the applied energy (i.e., intensity and/or duration), often ramping down soon after the start of stimulation. In the current study, we found that when using electrode configurations identical to the experimental condition, sensation differed significantly at one or more time points, both for current ramped down to 1.0 (active fade sham) and to zero (inert fade sham). Using a novel, active sham approach, which shunts a portion of the current through the scalp, we 
found no significant differences in scalp sensation ratings between the sham and experimental conditions when the current remained constant at $2 \mathrm{~mA}$; absence of differences has historically been the criteria for acceptance of a sham condition in previous research (e.g., Gandiga, Hummel, \& Cohen, 2006). However, it should be noted that this montage did not meet the more stringent criteria for statistical equivalence (though only exceeding MPD bounds by $<+/-.04$ at all time points) and resulted in greatly reduced, but perhaps not negligible, current induced in the cortex (see Figure 1b), the behavioral effects of which are unknown. Even with the variability of sensation ratings reported by our participants (a major limitation of investigations of such a subjective experience), it is clear that the inert fade sham condition is probably not the best candidate for blinding (i.e., significantly different, non-equivalent, consistently low sensation ratings across participants during zero current), particularly for crossover investigations where participants receive both sham and experimental conditions.

Given these results, other sham configurations that capitalize on scalp shunting of current and/or that match the scalp sensations of experimental conditions should be investigated further. For example, a proximal Sham $3 \times 1$ montage (see Figure 1c) should result in increased current shunting and comparable scalp sensations, and could be further explored as a viable sham. Alternatively, given that $1 \mathrm{~mA}$ resulted in floor or near floor sensation ratings for subjects in our pilot study, we feel it is plausible to reduce sensation in active/experimental conditions to negligible levels. This could be accomplished by applying current in parallel using HD electrode "functional sets" (e.g., splitting $2 \mathrm{~mA}$ across two adjacent center HD electrodes; see Figure 1d), which results in a comparable electric field to the experimental condition according to our model (compare with Figure 1a). If the target current were delivered in this manner, then sham development would be trivial and equivalence likely readily attained, as sensation ratings should be at or near floor for the active condition and require no active current flow for the sham condition.

In future investigations, attention should not only be paid to scalp sensations, but also to behavioral effects that could occur due to the small amount of current induced in the cortex. While modeling provides a best guess of how much current may have reached the cortex during these different conditions, an examination of the relationship between estimated electric field and physiological and/or behavioral relevance has not been clearly characterized and was not addressed in this study. In addition, future research should employ designs that carefully consider the pharmacokinetic properties of the local anesthetic used in scalp preparation. Because precise information about the half-life and duration of effect of benzocaine is unknown, we are unable to make post hoc inferences about the relationships between the amount of anesthetic applied, time post application, sensation ratings, and current delivery in this study.

This is the first NICS investigation to employ equivalence testing, and as such, we used rationale and criteria supported by related literature to determine the bounds of the MPD. Whether or not our strategy was too conservative to detect equivalence is unknown (but suspected) and future work should include development of standards for equivalence testing specific to NICS research in order to increase trial rigor in NICS research to ensure adequate sham development. Equivalence testing is a more rigorous approach that, if utilized, could prevent the continued development and use of NICS sham conditions that, though they do not result in significant differences, are still able to be detected by participants as different (and thus are not true shams). Our behavioral and modeling findings suggest that the flexibility of multi-electrode HD-tDCS should permit improvements in both active (reduced 
sensation) and sham conditions (equivalent sensation with negligible current) that will lead to enhanced quality and interpretability of NICS research.

\section{References}

Boggio, P., Sultani, N., Fecteau, S., Merabet, L., Mecca, T., Pascual-Leone, A., ... Fregni, F. (2008). Prefrontal cortex modulation using transcranial DC stimulation reduces alcohol craving: A double-blind, sham-controlled study. Drug and Alcohol Dependence, 92, 55-60. http://dx.doi.org/10.1016/j.drugalcdep.2007.06.011

Borckardt, J. J., Bikson, M., Frohman, H., Reeves, S. T., Datta, A., Bansal, V., ... George, M. S. (2012). A pilot study of the tolerability and effects of high-definition transcranial direct current stimulation on pain perception. Journal of Pain, 13, 112-120. http://dx.doi.org/10.1016/j.jpain.2011.07.001

Broadbent, H. J., van den Eynde, F., Guillaume, S., Hanif, E. L., Stahl, D., David, A. S., ... Schmidt, U. (2011). Blinding success of rTMS applied to the dorsolateral prefrontal cortex in randomized sham-controlled trials: A systematic review. World Journal of Biological Psychiatry, 12, 240-248. http://dx.doi.org/10.3109/15622975.2010.541281

Brunoni, A. R., Nitsche, M. A., Bolognini, N., Bikson, M., Wagner, T., Merabet, L., ... Fregni, F. (2012). Clinical research with transcranial direct current stimulation (t-DCS): Challenges and future direction. Brain Stimulation, 5, 175-195. http://dx.doi.org/10.1016/j.brs.2011.03.002

Chrysikou, E. G. \& Hamilton, R. H. (2011). Noninvasive brain stimulation in the treatment of aphasia: Exploring interhemispheric relationships and their implications for neurorehabilitation. Restorative Neurology and Neuroscience, 29, 375-394. http://dx.doi.org/10.3233/RNN-2011-0610

Coffman, B. A., Trumbo, M. C., \& Clark, V. P. (2012). Enhancement of object detection with transcranial direct current stimulation is associated with increased attention. BMC Neuroscience, 13, 108. http://dx.doi.org/10.1186/1471-2202-13-108

Datta, A., Bansal, V., Diaz, J., Patel, J., Reato, D., \& Bikson, M. (2009). Gyri-precise head model of transcranial direct current stimulation: Improved spatial focality using a ring electrode versus conventional rectangular pad. Brain Stimulation, 2, 201-207. http://dx.doi.org/ 10.1016/j.brs.2009.03.005

Deng, Z. D., \& Peterchev, A. V. (2011). Transcranial magnetic stimulation coil with electronically switchable active and sham modes. Conference Proceedings of the IEEE Engineering in Medicine and Biology Society, 1993-1996. http://dx.doi.org/10.1109/IEMBS.2011.6090561

Dmochowski, J. P., Datta, A., Bikson, M., Su, Y., \& Parra, L. C. (2011). Optimized multielectrode stimulation increases focality and intensity at target. Journal of Neural Engineering, 8, 046011. http://dx.doi.org/10.1088/1741-2560/8/4/046011 
Dundas, J. E., Thickbroom, G. W., \& Mastaglia, F. L. (2007). Perception of comfort during transcranial DC stimulation: Effect of $\mathrm{NaCl}$ solution concentration applied to sponge electrodes. Clinical Neurophysiology, 118, 1166-1170.

http://dx.doi.org/10.1016/j.clinph.2007.01.010

Fregni, F., \& Pascual-Leone, A. (2007). Technology insight: Nonivasive brain stimulation in neurology: Perspectives on the therapeutic potential of rTMS and tDCS. Nature Clinical Practice Neurology, 3, 383-393. http://dx.doi.org/10.1038/ncpneuro0530

Gandiga, P. C., Hummel, F. C., \& Cohen, L. G. (2006). Transcranial DC stimulation (tDCS): A tool for double-blind sham-controlled clinical studies in brain stimulation. Clinical Neurophysiology, 117, 845-850. http://dx.doi.org/10.1016/i.clinph.2005.12.003

Kessler, S. K., Turkeltaub, P. E., Benson, J. G., \& Hamilton, R. H. (2012). Differences in the experience of active and sham transcranial direct current stimulation. Brain Stimulation, 5, 155-162. http://dx.doi.org/10.1016/j.brs.2011.02.007

Loo, C. K., Taylor, J. L., Gandevia, S. C., McDarmont, B. N., Mitchell, P. B., \& Sachdev, P. S. (2000). Transcranial magnetic stimulation (TMS) in controlled treatment studies: Are some "sham" forms active? Biological Psychiatry, 47, 325-331. http://dx.doi.org/10.1016/S0006-3223(99)00285-1

Minhas, P., Bansal, V., Patel, J., Ho, J. S., Diaz, J., Datta, A., \& Bikson, M. (2010). Electrodes for high-definition transcutaneous DC stimulation for applications in drugdelivery and electrotherapy, including tDCS. Journal of Neuroscience Methods, 190, 188-197. http://dx.doi.org/10.1016/j.jneumeth.2010.05.007

Minhas, P., Datta, A., \& Bikson, M. (2011). Cutaneous perception during tDCS: Role of electrode shape and sponge salinity. Clinical Neurophysiology, 122, 637-638. http://dx.doi.org/10.1016/j.clinph.2010.09.023

Moncrieff, J., Wessely, S., \& Hardy, R. (2004). Active placebos versus antidepressants for depression. Cochrane Database of Systematic Reviews, 1. http://dx.doi.org/10.1002/14651858.CD003012.pub2

Rusticus, S., \& Lovato, C. Y. (2011). Applying tests of equivalence for multiple group comparisons: Demonstration of the confidence interval approach. Practical Assessment Research Evaluation, 16(7). http://pareonline.net/pdf/v16n7.pdf

Tryon, W. W. (2001). Evaluating statistical difference, equivalence, and indeterminacy using inferential confidence intervals: An integrated alternative method of conducting null hypothesis statistical tests. Psychological Methods, 6, 371-386. http://dx.doi.org/10.1037/1082-989X.6.4.371

Vallence, A-M. \& Ridding, M. C. (2013). Non-invasive induction of plasticity in the human cortex: Uses and limitations. Cortex. http://dx.doi.org/10.1016/j.cortex.2013.12.006

Villamar, M. F., Volz, M. S., Bikson, M., Datta, A., DaSilva, A. F., \& Fregni, F. (2013). Technique and considerations in the use of $4 \times 1$ ring high-definition transcranial direct current stimulation (HD-tDCS). Journal of Visualized Experiments, 77, e50309. http://dx.doi.org/10.3791/50309 
Williams, J. A., Imamura, M., \& Fregni, F. (2009). Updates on the use of non-invasive brain stimulation in physical and rehabilitation medicine. Journal of Rehabilitative Medicine, 41, 305-311. http://dx.doi.org/10.2340/16501977-0356

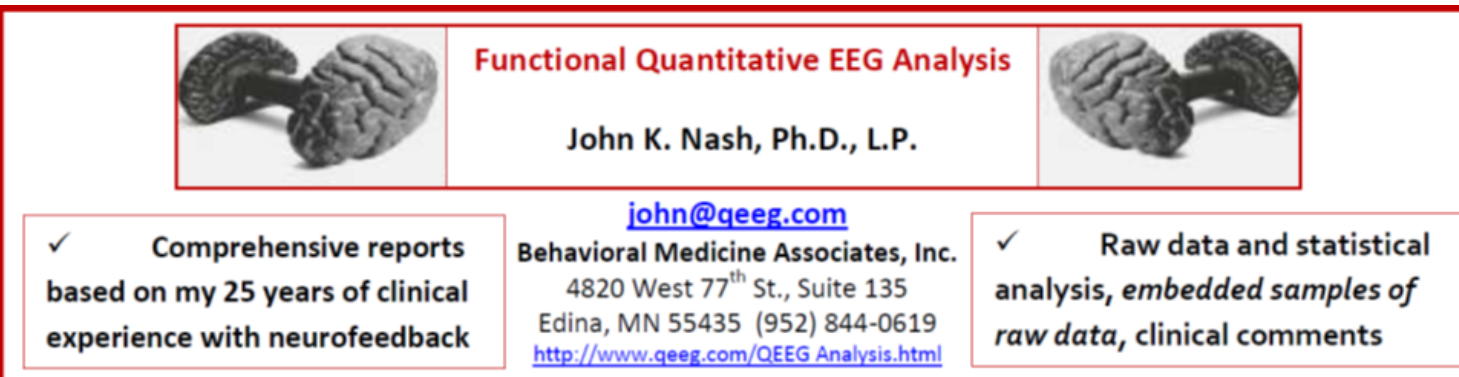

I've used neurofeedback in children, adolescents and adults, using 19 channel qEEG and normative reference databases since 1991, treating over 2000 patients. I've been involved with ISNR since its inception and have consulted on QEEG and neurofeedback for state government agencies, NASA, biomedical engineering firms, and on research at the University of MN and Mayo Clinic.

Functional QEEG: Consider recording an array of conditions to get the best understanding of the person and help avoid negative side-effects. Some people have abnormal findings in all conditions. Others have very normal EEGs during specific conditions, e.g. during Drawing the EEG may look very activated and normal ("art brain": teach these folks to use 'picture notes' to enhance attention during lectures). Using tasks allows you to see what tasks cause the greatest difficulty and gives you the data you need for a truly rational treatment plan. I recommend at a minimum you record eyes closed, eyes open, then reading and listening. A second eyes closed recording sometimes shows changes in alertness or anxiety. I do not examine the EEG for neurological disease.

$\rightarrow$ Basic Eyes Closed/Eyes Open analysis with the NeuroGuide Database and visual inspection of the raw data, $\mathbf{2 5 0}$

$\rightarrow$ Extended Analysis: Eyes Closed, Eyes Open and up to two other conditions (e.g., a $2^{\text {nd }}$ eyes closed and one active task), $\$ 350$

$\rightarrow$ Additional conditions: $\mathbf{\$ 2 5}$ for each additional task.

$\rightarrow$ E-mail or telephone consultation. $\mathbf{\$ 1 5 0 / h o u r ~ i n ~} 10$ minute units.

Client comment: "Thanks for such speedy turnaround time!!! The reports are indeed helpful." - Joy Lunt, RN 